\title{
STELLAR POPULATIONS IN THE PEGASUS DWARF GALAXY
}

\author{
MYUNG GYOON LEE \\ Department of Astronomy, Seoul National University \\ Seoul, 151-742, Korea; Email: mglee@astrog.snu.ac.kr
}

The Pegasus dwarf galaxy (DDO 216) is a resolved irregular galaxy. We present a study of stars in this galaxy based on $B V R I$ CCD photometry obtained using the Palomar $1.5 \mathrm{~m}$ telescope. The color-magnitude diagrams show (a) a dominant red giant branch (RGB) population, (b) a small number of asymptotic giant branch (AGB) population above the tip of the RGB (TRGB), and (c) a sparse popultion of massive young stars including the brightest red supergiants. The mean metallicity of the RGB has been estimated from the color of the RGB at $M_{I}=-3.5 \mathrm{mag}:[\mathrm{Fe} / \mathrm{H}]=-1.5 \pm 0.2 \mathrm{dex}$. The distance to this galaxy has been measured using the I-magnitude of the TRGB: $(m-M)_{0}=25.13 \pm 0.11$ $\operatorname{mag}(d=1060 \pm 50 \mathrm{kpc})$ (see Lee et al. 1993). This value is significantly smaller than the Cepheid distance estimate by Hoessel et al. (1990), $(m-M)_{0}=26.22 \pm 0.20(d=1750 \pm 160$ kpc). This large difference between the TRGB and Cepheid distance estimates are due to the fact that the variable stars considered as Cepheids by Hoessel et al. are not Cepheids, but other kinds of variable stars, as shown in Fig.1.

\section{References}

Hoessel, J. G., Abbot, M. J., Saha, A., Mossman, A. E., and Danielson, G. E. 1990, AJ, 100, 1151 Lee, M. G., Freedman, W. L., and Madore, B. F. 1993, ApJ, 417, 553

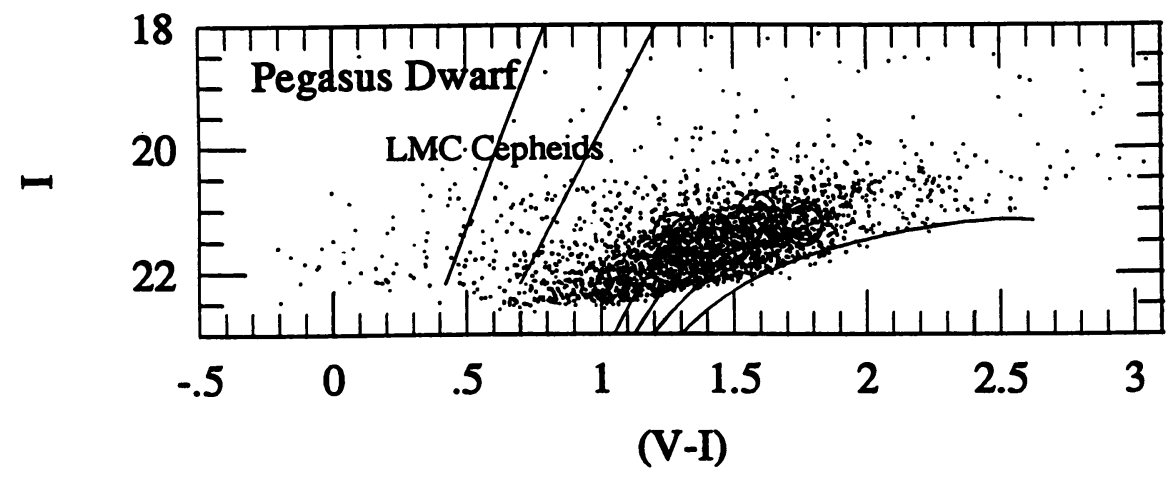

Figure 1. A comparison of the Pegasus dwarf galaxy with the red giant branches of the galactic globular clusters, M15 ([Fe/H] = -2.17), M2 $([\mathrm{Fe} / \mathrm{H}]=-1.58)$, NGC $1851([\mathrm{Fe} / \mathrm{H}]=-1.29)$, and $47 \mathrm{Tuc}([\mathrm{Fe} / \mathrm{H}]=$ -0.71) (curved solid lines). The open circles represent the very likely Cepheids and open triangles represent the probable Cepheids in Hoessel et al.'s study. Note that they are not located in the Cepheid instability strip, but in the RGB and AGB positions. 